\title{
Synthesis and Insilico Assessment of Novel Benzothiazole Series Against Human DNA Topo Isomerase I
}

\author{
Venkateshwarlu Lagishetty \\ Associate Professor \\ Department of Medicinal \\ Chemistry \\ Vikas College of Pharmacy, \\ Jangaon, Andhrapradesh, \\ India-506167
}

\author{
M. Sarangapani, PhD. \\ Professor, Department of Medicinal \\ Chemistry, University college of \\ Pharmaceutical sciences, \\ Kakatiya University, Warangal, \\ Andhrapradesh, India-506009
}

\author{
Sreenivas.Enaganti \\ Research associate, Asterace \\ Labs \\ 208, Ilnd floor, WindsorPlaza \\ Nallakunta, Hyderabad, \\ Andhra Pradesh, \\ India - 500044
}

\begin{abstract}
Human topoisomerase I (Top1) plays a pivotal role in cell replication and transcription, and therefore is an important anti-cancer target. Thiazolidinone and Azetidinone derivatives of 6-substituted 2-aminobenzothiazoles were synthesized and tested for their topoisomerase inhibitory activity and cytotoxicity by computational methods. Among the synthesized thiazolidinone and azetidinone analogs, compound 5avi and 6cvi exhibited potent topo I inhibitory activity and strong cytotoxicity. Interestingly, consistency was observed between the cytotoxicities and topo I activities in these thiazolidinone and azetidinone analogs, suggesting that the target of compound 5avi and 6cvi is limited to topo I. Molecular docking studies were performed with the Molecular Operating Environment (London DG Scoring Function) program to afford the ideal interaction mode of the compound into the binding site of the DNA-topo I complex in order to clarify the topo I activity of 5avi and 6cvi..
\end{abstract}

\section{Keywords}

Topoisomerase I, replication, transcription, cytoitoxicity, thiazolidene, azetidinone

\section{INTRODUCTION}

Eukaryotic DNA topoisomerase I (topo I) is a crucial enzyme that works to relax supercoiled DNA during replication, transcription, and mitosis [1]. In a number of human solid tumors, the intracellular level of topo $\mathrm{I}$ is higher than that in normal tissues, signifying that controlling the topo I level is essential in treating cancers [2]. By stabilizing the cleavable topo I-DNA ternary complex with drug, topo I inhibitors exhibit their antitumor activities. Therefore, topo I enzyme has been considered a promising target for the development of novel cancer chemotherapeutics [3]. Several reviews have compiled the numerous Top1 inhibitors that have been isolated, synthesized, and evaluated in vitro and in vivo $[4,5]$.

The X-ray crystal structure published by Stewart, et al. of the human DNA topoisomerase $(70 \mathrm{Kda})$ in complex with the camptothecin and covalent complex with A 22 base pair DNA duplex (PDB ID: 1T8I) were retrieved from protein data bank [6] based on good resolution and Ramachandran's plot analysis. The structure was chosen because of its high resolution $3.0 \AA$ compared to other available structures. In the Ramachandran's plot analysis, the residues were classified according to their regions in the quadrangle, which shows human DNA topoisomerase (1T8I) is having $87.3 \%$ residues in most favorable region and there is no residue in disallowed region.

In this investigation, we observed the chemical synthesis of benzylidene derivatives and their potent topo I inhibitory activity and cytotoxicity by docking using MOE software. In general, the binding mode of a drug to its receptor site is influenced by subtle electronic or steric factors, and these two functions play an important role in the bioactive conformation of the drug molecule. Rigid structures are commonly considered to have little conformational entropy compared to flexible molecules and can be more efficiently fitted into the binding site of a receptor [7].

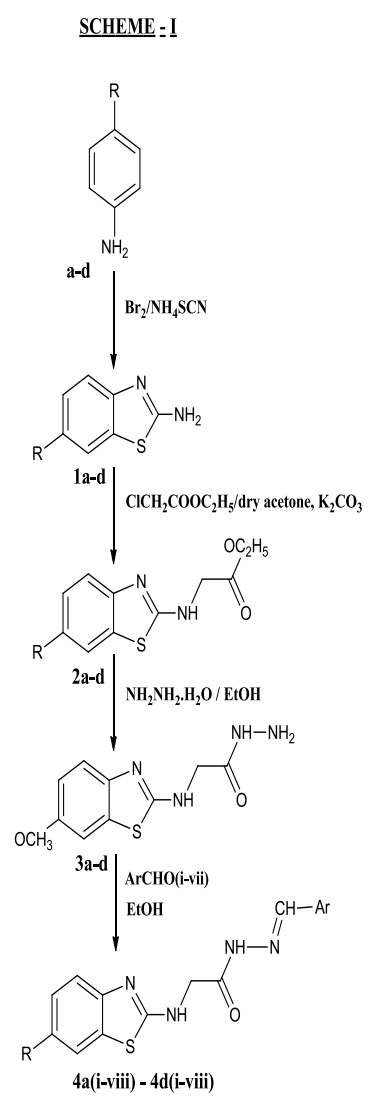




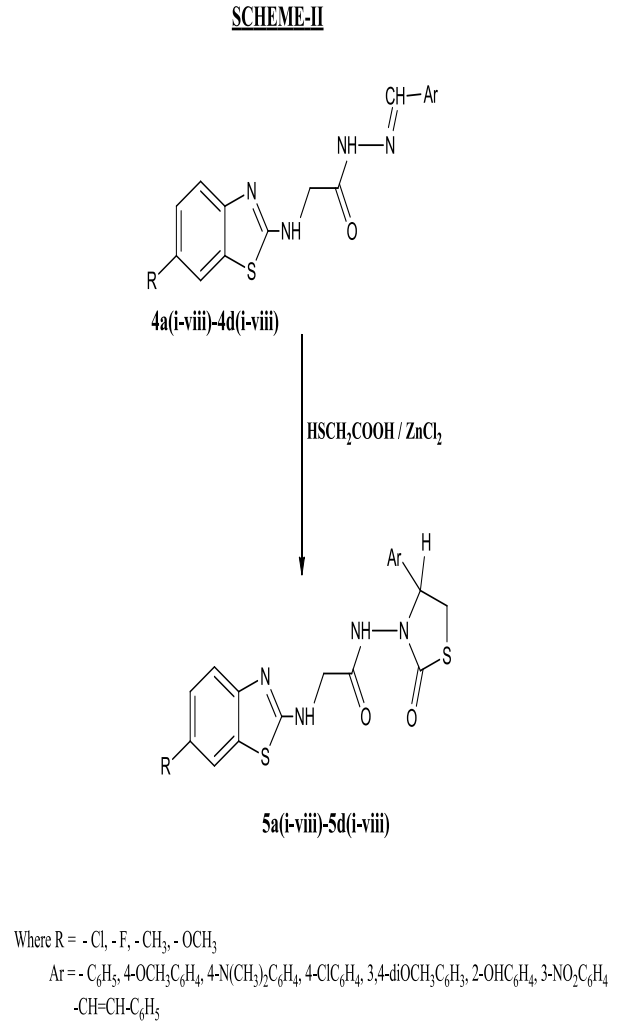

$\underline{\text { SCHEME-III }}$
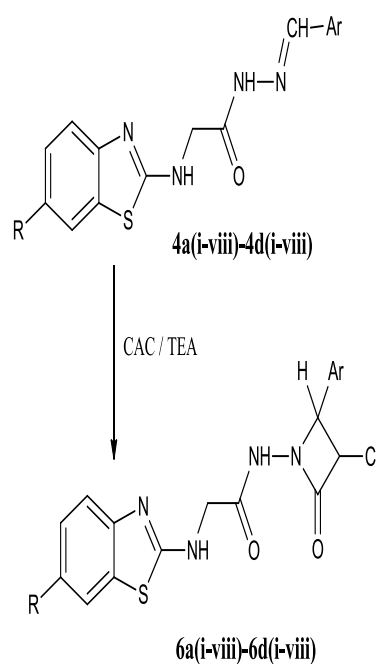

6a(i-viii)-6d(i-viii)

Where $\mathrm{R}=-\mathrm{Cl},-\mathrm{F},-\mathrm{CH}_{3},-\mathrm{OCH}_{3}$

$\left.\mathrm{Ar}=-\mathrm{C}_{6} \mathrm{H}_{5}, 4-0 \mathrm{CH}_{3} \mathrm{C}_{6} \mathrm{H}_{4}, 4 \mathrm{-}-\mathrm{N}_{\left(\mathrm{CH}_{3}\right.}\right)_{2} \mathrm{C}_{6} \mathrm{H}_{4}, 4-\mathrm{ClC}_{6} \mathrm{H}_{4}, 3,-4-\mathrm{HOCCH}_{3} \mathrm{C}_{6} \mathrm{H}_{3}, 2-\mathrm{OHC}_{6} \mathrm{H}_{4}, 3-\mathrm{NO}_{2} \mathrm{C}_{6} \mathrm{H}_{4}$ $-\mathrm{CH}=\mathrm{CH}_{-} \mathrm{C}_{6} \mathrm{H}_{5}$

\section{CHEMISTRY}

\subsection{Chemical synthesis of 6-substituted 2-} (benzo(d)thiazol-2ylamino)- $\mathrm{N}$-arylidene acetohydrazides $4 \mathbf{a}(\mathbf{i}$-viii) - $\mathbf{4 d}$ (i-viii):

An equimolar ( 0.01 mole) each mixture of $3 \mathrm{a}$ and appropriate aromatic aldehydes (i-viii) in ethanol $(25 \mathrm{ml})$ containing $2-3$ drops of acetic acid was refluxed on a water bath for 3-4hrs. The solvent was distilled off under reduced pressure and the residue was poured into ice cold water to obtain the product. The compound was filtered and washed with cold water and dried. The crude product was recrystallized from ethanol. Various arylidene derivatives were synthesized by adopting the procedure where the physical, analytical properties are cosidered

\subsection{Chemical synthesis of 6-substituted 2- (benzo(d)thiazol-2ylamino)-N-(2-aryl-4- oxothiazolidine-3-yl) acetamide 5a(i-viii) - 5d(i-viii):}

A mixture of 6-substituted 2-(benzo(d)thiazol-2ylamino)-Narylidene acetohydrazides [4a(i-viii) - 4d(i-viii); 0.001 mole] and mercaptoacetic acid (0.001 mole) was dissolved in dioxane $(20 \mathrm{ml})$ and pinch of anhydrous zinc chloride was added. The reaction mixture was heated under reflux for 12 hrs. and the solvent was removed as far as possible. The residue was cooled triturated with crushed ice $(50 \mathrm{gm})$. The solid separated was filtered, washed with $5 \%$ sodium bicarbonate solution until no effervescence were observed and then with portion of a cold water. The crude product was purified by recrystallisation from ethanol to get a crystalline compound.

Following the general procedure described above, thiazolidinones $5 \mathrm{a}$ (i-viii) $-5 \mathrm{~d}(\mathrm{i}$-viii) were synthesized and characterized data's are given in the Table-I.

\subsection{Chemical synthesis of 2 -(benzo(d) thiazol-2ylamino)- $\mathrm{N}$-(4-aryl-3-chloro-2-oxo azetidin-1-yl)acetamide[6a(i-viii)-6d(i-viii)]:}

To a mixture of 6-substituted 2-(benzo(d)thiazol-2ylamino)$\mathrm{N}$-arylidene acetohydrazides [4a(i-viii) - 4d(i-viii); 0.001 mole], triethylamine (0.003 mole) dissolved in dioxane (25 $\mathrm{ml})$ and chloroacetylchloride $(0.0012$ mole) was added drop wise while cooling and stirring. The reaction mixture was stirred for $14 \mathrm{hrs}$. at room temperature and solvent was removed under reduced pressure. The residue was cooled and triturated with crushed ice (50 gm). The solid separated was filtered, washed with small portion of cold water and dried. The product was purified by recrystallisation from aqueous ethanol to get a pure crystalline compound.

Adopting the similar procedure the azetidinones were synthesized whose physical and analytical particulars are given in Table-II. 
Table-I: Thiazolidinone series: Physical and analytical data of 6-substituted 2-(benzo(d)thiazol-2ylamino)-N-(2-aryl-4oxothiazolidine-3-yl) acetamide [5a(i-viii) - 5d(i-viii)]

\begin{tabular}{|c|c|c|c|c|c|c|c|c|c|c|}
\hline \multirow[t]{2}{*}{$\begin{array}{l}. \mathrm{N} \\
\mathrm{o}\end{array}$} & \multirow[t]{2}{*}{ Series } & \multirow[t]{2}{*}{$\mathrm{R}$} & \multirow[t]{2}{*}{$\mathrm{Ar}$} & \multirow[t]{2}{*}{$\begin{array}{l}\text { Molecular } \\
\text { Formula }\end{array}$} & \multirow[t]{2}{*}{$\begin{array}{l}\text { Mol. } \\
\text { Wt. }\end{array}$} & \multirow[t]{2}{*}{$\begin{array}{l}\text { m.p. } \\
\left({ }^{\circ} \mathrm{C}\right)\end{array}$} & \multirow[t]{2}{*}{$\begin{array}{l}\text { Yield } \\
(\%)\end{array}$} & \multicolumn{3}{|c|}{$\begin{array}{l}\text { Elemental analyses } \\
\text { Calculated (Found) }\end{array}$} \\
\hline & & & & & & & & C & $\mathbf{H}$ & $\mathbf{N}$ \\
\hline 1 & 5ai & $-\mathrm{Cl}$ & $-\mathrm{C}_{6} \mathrm{H}_{5}$ & $\mathrm{C}_{18} \mathrm{H}_{15} \mathrm{ClN}_{4} \mathrm{O}_{2} \mathrm{~S}_{2}$ & 418 & 155 & 90 & 51.61 & 3.61 & 13.37 \\
\hline 2 & 5aii & $-\mathrm{Cl}$ & $-4-\mathrm{OCH}_{3} \mathrm{C}_{6} \mathrm{H}_{4}$ & $\mathrm{C}_{19} \mathrm{H}_{17} \mathrm{ClN}_{4} \mathrm{O}_{3} \mathrm{~S}_{2}$ & 448 & 173 & 76 & 50.63 & 3.82 & 12.48 \\
\hline 3 & 5aiii & $-\mathrm{Cl}$ & $-4-\mathrm{N}\left(\mathrm{CH}_{3}\right)_{2} \mathrm{C}_{6} \mathrm{H}_{4}$ & $\mathrm{C}_{20} \mathrm{H}_{20} \mathrm{ClN}_{5} \mathrm{O}_{2} \mathrm{~S}_{2}$ & 461 & 148 & 80 & 52.00 & 4.36 & 15.16 \\
\hline 4 & 5aiv & $-\mathrm{Cl}$ & $-4-\mathrm{ClC}_{6} \mathrm{H}_{4}$ & $\mathrm{C}_{18} \mathrm{H}_{14} \mathrm{Cl}_{2} \mathrm{~N}_{4} \mathrm{O}_{2} \mathrm{~S}_{2}$ & 453 & 144 & 65 & 47.69 & 3.11 & 12.36 \\
\hline 5 & $5 \mathrm{av}$ & $-\mathrm{Cl}$ & $-3,4-d i \mathrm{OCH}_{3} \mathrm{C}_{6} \mathrm{H}_{3}$ & $\mathrm{C}_{20} \mathrm{H}_{19} \mathrm{ClN}_{4} \mathrm{O}_{4} \mathrm{~S}_{2}$ & 478 & 165 & 60 & 50.15 & 4.00 & 11.70 \\
\hline 6 & 5avi & $-\mathrm{Cl}$ & $-2-\mathrm{OH} \mathrm{C}{ }_{6} \mathrm{H}_{4}$ & $\mathrm{C}_{18} \mathrm{H}_{15} \mathrm{ClN}_{4} \mathrm{O}_{3} \mathrm{~S}_{2}$ & 434 & 146 & 82 & 49.71 & 3.48 & 12.88 \\
\hline 7 & 5avii & $-\mathrm{Cl}$ & $-3-\mathrm{NO}_{2} \mathrm{C}_{6} \mathrm{H}_{4}$ & $\mathrm{C}_{18} \mathrm{H}_{14} \mathrm{ClN}_{5} \mathrm{O}_{4} \mathrm{~S}_{2}$ & 463 & 122 & 68 & 46.60 & 3.04 & 15.10 \\
\hline 8 & 5aviii & $-\mathrm{Cl}$ & $-\mathrm{CH}=\mathrm{CH}-\mathrm{C}_{6} \mathrm{H}_{5}$ & $\mathrm{C}_{20} \mathrm{H}_{17} \mathrm{ClN}_{4} \mathrm{O}_{2} \mathrm{~S}_{2}$ & 444 & 158 & 84 & 53.99 & 3.85 & 12.59 \\
\hline 9 & $5 \mathrm{bi}$ & $-\mathrm{F}$ & $-\mathrm{C}_{6} \mathrm{H}_{5}$ & $\mathrm{C}_{18} \mathrm{H}_{15} \mathrm{FN}_{4} \mathrm{O}_{2} \mathrm{~S}_{2}$ & 402 & 140 & 65 & 53.72 & 3.76 & 13.92 \\
\hline 10 & 5 bii & $-\mathrm{F}$ & $-4-\mathrm{OCH}_{3} \mathrm{C}_{6} \mathrm{H}_{4}$ & $\mathrm{C}_{19} \mathrm{H}_{17} \mathrm{FN}_{4} \mathrm{O}_{3} \mathrm{~S}_{2}$ & 432 & 164 & 82 & 52.76 & 3.96 & 12.95 \\
\hline 11 & 5biii & $-\mathrm{F}$ & $-4-\mathrm{N}\left(\mathrm{CH}_{3}\right)_{2} \mathrm{C}_{6} \mathrm{H}_{4}$ & $\mathrm{C}_{20} \mathrm{H}_{20} \mathrm{FN}_{5} \mathrm{O}_{2} \mathrm{~S}_{2}$ & 445 & 146 & 76 & 53.92 & 4.52 & 15.72 \\
\hline 12 & 5biv & $-\mathrm{F}$ & $-4-\mathrm{ClC}_{6} \mathrm{H}_{4}$ & $\mathrm{C}_{18} \mathrm{H}_{14} \mathrm{ClFN}_{4} \mathrm{O}_{2} \mathrm{~S}_{2}$ & 436 & 148 & 56 & 49.48 & 3.23 & 12.82 \\
\hline 13 & $5 \mathrm{bv}$ & $-F$ & $-3,4-d i \mathrm{OCH}_{3} \mathrm{C}_{6} \mathrm{H}_{3}$ & $\mathrm{C}_{20} \mathrm{H}_{19} \mathrm{FN}_{4} \mathrm{O}_{4} \mathrm{~S}_{2}$ & 462 & 140 & 80 & 51.94 & 4.14 & 12.11 \\
\hline 14 & 5 bvi & $-\mathrm{F}$ & $-2-\mathrm{OH} \mathrm{C}{ }_{6} \mathrm{H}_{4}$ & $\mathrm{C}_{18} \mathrm{H}_{15} \mathrm{FN}_{4} \mathrm{O}_{3} \mathrm{~S}_{2}$ & 418 & 156 & 75 & 51.66 & 3.61 & 13.39 \\
\hline 15 & 5 bvii & $-\mathrm{F}$ & $-3-\mathrm{NO}_{2} \mathrm{C}_{6} \mathrm{H}_{4}$ & $\mathrm{C}_{18} \mathrm{H}_{14} \mathrm{FN}_{5} \mathrm{O}_{4} \mathrm{~S}_{2}$ & 447 & 154 & 70 & 48.32 & 3.15 & 15.65 \\
\hline 16 & 5bviii & $-\mathrm{F}$ & $-\mathrm{CH}=\mathrm{CH}-\mathrm{C}_{6} \mathrm{H}_{5}$ & $\mathrm{C}_{20} \mathrm{H}_{17} \mathrm{FN}_{4} \mathrm{O}_{2} \mathrm{~S}_{2}$ & 428 & 176 & 68 & 56.06 & 4.00 & 13.08 \\
\hline 17 & $5 \mathrm{ci}$ & $-\mathrm{CH}_{3}$ & $-\mathrm{C}_{6} \mathrm{H}_{5}$ & $\mathrm{C}_{19} \mathrm{H}_{18} \mathrm{~N}_{4} \mathrm{O}_{2} \mathrm{~S}_{2}$ & 398 & 240 & 82 & 57.27 & 4.55 & 14.06 \\
\hline 18 & $5 \mathrm{cii}$ & $-\mathrm{CH}_{3}$ & $-4-\mathrm{OCH}_{3} \mathrm{C}_{6} \mathrm{H}_{4}$ & $\mathrm{C}_{20} \mathrm{H}_{20} \mathrm{~N}_{4} \mathrm{O}_{3} \mathrm{~S}_{2}$ & 428 & 262 & 56 & 56.06 & 4.70 & 13.07 \\
\hline 19 & 5 ciii & $-\mathrm{CH}_{3}$ & $-4-\mathrm{N}\left(\mathrm{CH}_{3}\right)_{2} \mathrm{C}_{6} \mathrm{H}_{4}$ & $\mathrm{C}_{21} \mathrm{H}_{23} \mathrm{~N}_{5} \mathrm{O}_{2} \mathrm{~S}_{2}$ & 441 & 244 & 78 & 57.12 & 5.25 & 15.86 \\
\hline 20 & 5 civ & $-\mathrm{CH}_{3}$ & $-4-\mathrm{ClC}_{6} \mathrm{H}_{4}$ & $\mathrm{C}_{19} \mathrm{H}_{17} \mathrm{ClN}_{4} \mathrm{O}_{2} \mathrm{~S}_{2}$ & 432 & 262 & 74 & 52.71 & 3.96 & 12.94 \\
\hline 21 & $5 \mathrm{cv}$ & $-\mathrm{CH}_{3}$ & $-3,4-d i \mathrm{OCH}_{3} \mathrm{C}_{6} \mathrm{H}_{3}$ & $\mathrm{C}_{21} \mathrm{H}_{22} \mathrm{~N}_{4} \mathrm{O}_{4} \mathrm{~S}_{2}$ & 458 & 250 & 64 & 55.00 & 4.84 & 12.22 \\
\hline 22 & $5 \mathrm{cvi}$ & $-\mathrm{CH}_{3}$ & $-2-\mathrm{OH} \mathrm{C}{ }_{6} \mathrm{H}_{4}$ & $\mathrm{C}_{19} \mathrm{H}_{18} \mathrm{~N}_{4} \mathrm{O}_{3} \mathrm{~S}_{2}$ & 414 & 272 & 72 & 50.05 & 4.38 & 13.52 \\
\hline 23 & 5 cvii & $-\mathrm{CH}_{3}$ & $-3-\mathrm{NO}_{2} \mathrm{C}_{6} \mathrm{H}_{4}$ & $\mathrm{C}_{19} \mathrm{H}_{17} \mathrm{~N}_{5} \mathrm{O}_{4} \mathrm{~S}_{2}$ & 443 & 258 & 78 & 51.46 & 3.86 & 15.79 \\
\hline 24 & 5cviii & $-\mathrm{CH}_{3}$ & $-\mathrm{CH}=\mathrm{CH}-\mathrm{C}_{6} \mathrm{H}_{5}$ & $\mathrm{C}_{21} \mathrm{H}_{20} \mathrm{~N}_{4} \mathrm{O}_{2} \mathrm{~S}_{2}$ & 424 & 256 & 64 & 59.41 & 4.75 & 13.20 \\
\hline 25 & $5 \mathrm{di}$ & $-\mathrm{OCH}_{3}$ & $-\mathrm{C}_{6} \mathrm{H}_{5}$ & $\mathrm{C}_{19} \mathrm{H}_{18} \mathrm{~N}_{4} \mathrm{O}_{3} \mathrm{~S}_{2}$ & 414 & 240 & 72 & 55.05 & 4.38 & 13.52 \\
\hline 26 & $5 \mathrm{dii}$ & $-\mathrm{OCH}_{3}$ & $-4-\mathrm{OCH}_{3} \mathrm{C}_{6} \mathrm{H}_{4}$ & $\mathrm{C}_{20} \mathrm{H}_{20} \mathrm{~N}_{4} \mathrm{O}_{4} \mathrm{~S} 2$ & 444 & 252 & 65 & 54.04 & 4.53 & 12.60 \\
\hline 27 & 5diii & $-\mathrm{OCH}_{3}$ & $-4-\mathrm{N}\left(\mathrm{CH}_{3}\right)_{2} \mathrm{C}_{6} \mathrm{H}_{4}$ & $\mathrm{C}_{21} \mathrm{H}_{23} \mathrm{~N}_{5} \mathrm{O}_{3} \mathrm{~S}_{2}$ & 457 & 234 & 60 & 55.12 & 5.07 & 15.31 \\
\hline 28 & $5 \mathrm{div}$ & $-\mathrm{OCH}_{3}$ & $-4-\mathrm{ClC}_{6} \mathrm{H}_{4}$ & $\mathrm{C}_{19} \mathrm{H}_{17} \mathrm{ClN}_{4} \mathrm{O}_{3} \mathrm{~S}_{2}$ & 448 & 230 & 75 & 50.83 & 3.82 & 12.48 \\
\hline 29 & $5 \mathrm{dv}$ & $-\mathrm{OCH}_{3}$ & $-3,4-d i \mathrm{OCH}_{3} \mathrm{C}_{6} \mathrm{H}_{3}$ & $\mathrm{C}_{21} \mathrm{H}_{22} \mathrm{~N}_{4} \mathrm{O}_{5} \mathrm{~S}_{2}$ & 474 & 224 & 65 & 53.15 & 4.67 & 11.81 \\
\hline 30 & $5 \mathrm{dvi}$ & $-\mathrm{OCH}_{3}$ & $-2-\mathrm{OH} \mathrm{C}{ }_{6} \mathrm{H}_{4}$ & $\mathrm{C}_{19} \mathrm{H}_{18} \mathrm{~N}_{4} \mathrm{O}_{4} \mathrm{~S}_{2}$ & 430 & 222 & 70 & 53.01 & 4.21 & 13.01 \\
\hline 31 & $5 \mathrm{dvii}$ & $-\mathrm{OCH}_{3}$ & $-3-\mathrm{NO}_{2} \mathrm{C}_{6} \mathrm{H}_{4}$ & $\mathrm{C}_{19} \mathrm{H}_{17} \mathrm{~N}_{5} \mathrm{O}_{5} \mathrm{~S}_{2}$ & 459 & 238 & 83 & 49.66 & 3.73 & 15.24 \\
\hline 32 & 5dviii & $-\mathrm{OCH}_{3}$ & $-\mathrm{CH}=\mathrm{CH}-\mathrm{C}_{6} \mathrm{H}_{5}$ & $\mathrm{C}_{21} \mathrm{H}_{20} \mathrm{~N}_{4} \mathrm{O}_{3} \mathrm{~S}_{2}$ & 440 & 246 & 64 & 57.25 & 4.58 & 12.72 \\
\hline
\end{tabular}


Table II: Azetidinone series Table-III: Physical and analytical data of 2-(benzo(d)thiazol-2ylamino)-N-(4-aryl-3-chloro-2oxoazetidin-1-yl) acetamide [6a(i-viii) - 6d(i-viii)]

\begin{tabular}{|c|c|c|c|c|c|c|c|c|c|c|}
\hline \multirow[t]{2}{*}{ S.No. } & \multirow[t]{2}{*}{ Series } & \multirow[t]{2}{*}{$\mathrm{R}$} & \multirow[t]{2}{*}{$\mathrm{Ar}$} & \multirow[t]{2}{*}{$\begin{array}{l}\text { Molecular } \\
\text { Formula }\end{array}$} & \multirow[t]{2}{*}{$\begin{array}{l}\text { Mol. } \\
\text { Wt. }\end{array}$} & \multirow[t]{2}{*}{$\begin{array}{l}\text { m.p. } \\
\left({ }^{\circ} \mathrm{C}\right)\end{array}$} & \multirow[t]{2}{*}{$\begin{array}{l}\text { Yield } \\
(\%)\end{array}$} & \multicolumn{3}{|c|}{$\begin{array}{l}\text { Elemental analyses } \\
\text { Calculated }\end{array}$} \\
\hline & & & & & & & & $\mathbf{C}$ & $\mathbf{H}$ & $\mathbf{N}$ \\
\hline 1 & 6ai & $-\mathrm{Cl}$ & $-\mathrm{C}_{6} \mathrm{H}_{5}$ & $\mathrm{C}_{18} \mathrm{H}_{14} \mathrm{Cl}_{2} \mathrm{~N}_{4} \mathrm{O}_{2} \mathrm{~S}$ & 421 & 190 & 70 & 51.32 & 3.35 & 13.30 \\
\hline 2 & 6aii & $-\mathrm{Cl}$ & $-4-\mathrm{OCH}_{3} \mathrm{C}_{6} \mathrm{H}_{4}$ & $\mathrm{C}_{19} \mathrm{H}_{16} \mathrm{Cl}_{2} \mathrm{~N}_{4} \mathrm{O}_{3} \mathrm{~S}$ & 451 & 184 & 34 & 50.56 & 3.57 & 12.41 \\
\hline 3 & 6aiii & $-\mathrm{Cl}$ & $-4-\mathrm{N}\left(\mathrm{CH}_{3}\right)_{2} \mathrm{C}_{6} \mathrm{H}_{4}$ & $\mathrm{C}_{20} \mathrm{H}_{19} \mathrm{Cl}_{2} \mathrm{~N}_{5} \mathrm{O}_{2} \mathrm{~S}$ & 464 & 178 & 88 & 51.73 & 4.12 & 15.08 \\
\hline 4 & 6aiv & $-\mathrm{Cl}$ & $-4-\mathrm{ClC}_{6} \mathrm{H}_{4}$ & $\mathrm{C}_{18} \mathrm{H}_{13} \mathrm{Cl}_{3} \mathrm{~N}_{4} \mathrm{O}_{2} \mathrm{~S}$ & 455 & 152 & 64 & 47.44 & 2.88 & 12.29 \\
\hline 5 & 6av & $-\mathrm{Cl}$ & $-3,4-d i \mathrm{OCH}_{3} \mathrm{C}_{6} \mathrm{H}_{3}$ & $\mathrm{C}_{20} \mathrm{H}_{18} \mathrm{Cl}_{2} \mathrm{~N}_{4} \mathrm{O}_{4} \mathrm{~S}$ & 481 & 186 & 69 & 49.90 & 3.77 & 11.64 \\
\hline 6 & 6avi & $-\mathrm{Cl}$ & $-2-\mathrm{OH} \mathrm{C}{ }_{6} \mathrm{H}_{4}$ & $\mathrm{C}_{18} \mathrm{H}_{14} \mathrm{Cl}_{2} \mathrm{~N}_{4} \mathrm{O}_{3} \mathrm{~S}$ & 437 & 180 & 78 & 49.44 & 3.23 & 12.81 \\
\hline 7 & 6avii & $-\mathrm{Cl}$ & $-3-\mathrm{NO}_{2} \mathrm{C}_{6} \mathrm{H}_{4}$ & $\mathrm{C}_{18} \mathrm{H}_{13} \mathrm{Cl}_{2} \mathrm{~N}_{5} \mathrm{O}_{4} \mathrm{~S}$ & 466 & 115 & 74 & 46.36 & 2.81 & 15.02 \\
\hline 8 & 6aviii & $-\mathrm{Cl}$ & $-\mathrm{CH}=\mathrm{CH}-\mathrm{C}_{6} \mathrm{H}_{5}$ & $\mathrm{C}_{20} \mathrm{H}_{16} \mathrm{Cl}_{2} \mathrm{~N}_{4} \mathrm{O}_{2} \mathrm{~S}$ & 447 & 194 & 64 & 53.70 & 3.61 & 12.32 \\
\hline 9 & $6 b i$ & $-\mathrm{F}$ & $-\mathrm{C}_{6} \mathrm{H}_{5}$ & $\mathrm{C}_{18} \mathrm{H}_{14} \mathrm{ClFN}_{4} \mathrm{O}_{2} \mathrm{~S}$ & 404 & 174 & 83 & 53.40 & 3.49 & 13.84 \\
\hline 10 & 6 bii & $-\mathrm{F}$ & $-4-\mathrm{OCH}_{3} \mathrm{C}_{6} \mathrm{H}_{4}$ & $\mathrm{C}_{19} \mathrm{H}_{16} \mathrm{ClFN}_{4} \mathrm{O}_{3} \mathrm{~S}$ & 434 & 168 & 78 & 52.48 & 3.71 & 12.88 \\
\hline 11 & 6biii & $-\mathrm{F}$ & $-4-\mathrm{N}\left(\mathrm{CH}_{3}\right)_{2} \mathrm{C}_{6} \mathrm{H}_{4}$ & $\mathrm{C}_{20} \mathrm{H}_{19} \mathrm{ClFN}_{5} \mathrm{O}_{2} \mathrm{~S}$ & 447 & 172 & 91 & 53.63 & 4.28 & 15.64 \\
\hline 12 & 6biv & $-\mathrm{F}$ & $-4-\mathrm{ClC}_{6} \mathrm{H}_{4}$ & $\mathrm{C}_{18} \mathrm{H}_{13} \mathrm{Cl}_{2} \mathrm{FN}_{4} \mathrm{O}_{2} \mathrm{~S}$ & 439 & 136 & 86 & 49.21 & 2.98 & 12.75 \\
\hline 13 & $6 b v$ & $-F$ & $-3,4-d i \mathrm{OCH}_{3} \mathrm{C}_{6} \mathrm{H}_{3}$ & $\mathrm{C}_{20} \mathrm{H}_{18} \mathrm{ClFN}_{4} \mathrm{O}_{4} \mathrm{~S}$ & 464 & 166 & 81 & 51.67 & 3.90 & 12.05 \\
\hline 14 & 6bvi & $-\mathrm{F}$ & $-2-\mathrm{OH} \mathrm{C}{ }_{6} \mathrm{H}_{4}$ & $\mathrm{C}_{18} \mathrm{H}_{14} \mathrm{ClFN}_{4} \mathrm{O}_{3} \mathrm{~S}$ & 420 & 185 & 74 & 51.37 & 3.35 & 13.31 \\
\hline 15 & 6bvii & $-\mathrm{F}$ & $-3-\mathrm{NO}_{2} \mathrm{C}_{6} \mathrm{H}_{4}$ & $\mathrm{C}_{18} \mathrm{H}_{13} \mathrm{ClFN}_{5} \mathrm{O}_{4} \mathrm{~S}$ & 449 & 176 & 95 & 48.06 & 2.91 & 15.57 \\
\hline 16 & 6bviii & $-\mathrm{F}$ & $-\mathrm{CH}=\mathrm{CH}-\mathrm{C}_{6} \mathrm{H}_{5}$ & $\mathrm{C}_{20} \mathrm{H}_{16} \mathrm{ClFN}_{4} \mathrm{O}_{2} \mathrm{~S}$ & 430 & 124 & 64 & 55.75 & 3.74 & 13.00 \\
\hline 17 & $6 \mathrm{ci}$ & $-\mathrm{CH}_{3}$ & $-\mathrm{C}_{6} \mathrm{H}_{5}$ & $\mathrm{C}_{19} \mathrm{H}_{17} \mathrm{ClN}_{4} \mathrm{O}_{2} \mathrm{~S}$ & 400 & 220 & 96 & 56.93 & 4.27 & 13.98 \\
\hline 18 & 6 cii & $-\mathrm{CH}_{3}$ & $-4-\mathrm{OCH}_{3} \mathrm{C}_{6} \mathrm{H}_{4}$ & $\mathrm{C}_{20} \mathrm{H}_{19} \mathrm{ClN}_{4} \mathrm{O}_{3} \mathrm{~S}$ & 430 & 270 & 89 & 55.75 & 4.44 & 13.00 \\
\hline 19 & 6ciii & $-\mathrm{CH}_{3}$ & $-4-\mathrm{N}\left(\mathrm{CH}_{3}\right)_{2} \mathrm{C}_{6} \mathrm{H}_{4}$ & $\mathrm{C}_{21} \mathrm{H}_{22} \mathrm{ClN}_{5} \mathrm{O}_{2} \mathrm{~S}$ & 443 & 276 & 85 & 56.81 & 4.99 & 15.78 \\
\hline 20 & $6 \operatorname{civ}$ & $-\mathrm{CH}_{3}$ & $-4-\mathrm{ClC}_{6} \mathrm{H}_{4}$ & $\mathrm{C}_{19} \mathrm{H}_{16} \mathrm{Cl}_{2} \mathrm{~N}_{4} \mathrm{O}_{2} \mathrm{~S}$ & 435 & 246 & 81 & 52.42 & 3.70 & 12.87 \\
\hline 21 & $6 \mathrm{cv}$ & $-\mathrm{CH}_{3}$ & $-3,4-d i \mathrm{OCH}_{3} \mathrm{C}_{6} \mathrm{H}_{3}$ & $\mathrm{C}_{21} \mathrm{H}_{21} \mathrm{ClN}_{4} \mathrm{O}_{4} \mathrm{~S}$ & 460 & 264 & 85 & 54.72 & 4.59 & 12.16 \\
\hline 22 & $6 \mathrm{cvi}$ & $-\mathrm{CH}_{3}$ & $-2-\mathrm{OH} \mathrm{C}{ }_{6} \mathrm{H}_{4}$ & $\mathrm{C}_{19} \mathrm{H}_{17} \mathrm{ClN}_{4} \mathrm{O}_{3} \mathrm{~S}$ & 416 & 280 & 67 & 54.74 & 4.11 & 13.44 \\
\hline 23 & 6 cvii & $-\mathrm{CH}_{3}$ & $-3-\mathrm{NO}_{2} \mathrm{C}_{6} \mathrm{H}_{4}$ & $\mathrm{C}_{19} \mathrm{H}_{16} \mathrm{ClN}_{5} \mathrm{O}_{4} \mathrm{~S}$ & 445 & 286 & 71 & 51.18 & 3.62 & 15.71 \\
\hline 24 & 6cviii & $-\mathrm{CH}_{3}$ & $-\mathrm{CH}=\mathrm{CH}-\mathrm{C}_{6} \mathrm{H}_{5}$ & $\mathrm{C}_{21} \mathrm{H}_{19} \mathrm{ClN}_{4} \mathrm{O}_{2} \mathrm{~S}$ & 426 & 160 & 76 & 59.08 & 4.49 & 13.12 \\
\hline 25 & $6 \mathrm{di}$ & $-\mathrm{OCH}_{3}$ & $-\mathrm{C}_{6} \mathrm{H}_{5}$ & $\mathrm{C}_{19} \mathrm{H}_{17} \mathrm{ClN}_{4} \mathrm{O}_{3} \mathrm{~S}$ & 416 & 130 & 74 & 54.74 & 4.11 & 13.44 \\
\hline 26 & 6dii & $-\mathrm{OCH}_{3}$ & $-4-\mathrm{OCH}_{3} \mathrm{C}_{6} \mathrm{H}_{4}$ & $\mathrm{C}_{20} \mathrm{H}_{19} \mathrm{ClN}_{4} \mathrm{O}_{4} \mathrm{~S}$ & 446 & 122 & 66 & 53.79 & 4.29 & 12.54 \\
\hline 27 & 6diii & $-\mathrm{OCH}_{3}$ & $-4-\mathrm{N}\left(\mathrm{CH}_{3}\right)_{2} \mathrm{C}_{6} \mathrm{H}_{4}$ & $\mathrm{C}_{21} \mathrm{H}_{22} \mathrm{ClN}_{5} \mathrm{O}_{3} \mathrm{~S}$ & 459 & 160 & 70 & 54.84 & 4.82 & 15.23 \\
\hline 28 & $6 \operatorname{div}$ & $-\mathrm{OCH}_{3}$ & $-4-\mathrm{ClC}_{6} \mathrm{H}_{4}$ & $\mathrm{C}_{19} \mathrm{H}_{16} \mathrm{Cl}_{2} \mathrm{~N}_{4} \mathrm{O}_{3} \mathrm{~S}$ & 451 & 152 & 56 & 50.56 & 3.57 & 12.41 \\
\hline 29 & $6 \mathrm{dv}$ & $-\mathrm{OCH}_{3}$ & $-3,4-d i \mathrm{OCH}_{3} \mathrm{C}_{6} \mathrm{H}_{3}$ & $\mathrm{C}_{21} \mathrm{H}_{21} \mathrm{ClN}_{4} \mathrm{O}_{5} \mathrm{~S}$ & 476 & 114 & 53 & 52.88 & 4.44 & 11.75 \\
\hline 30 & $6 \mathrm{dvi}$ & $-\mathrm{OCH}_{3}$ & $-2-\mathrm{OH} \mathrm{C} \mathrm{H}_{4}$ & $\mathrm{C}_{19} \mathrm{H}_{17} \mathrm{ClN}_{4} \mathrm{O}_{4} \mathrm{~S}$ & 432 & 98 & 60 & 52.72 & 3.96 & 12.94 \\
\hline 31 & 6dvii & $-\mathrm{OCH}_{3}$ & $-3-\mathrm{NO}_{2} \mathrm{C}_{6} \mathrm{H}_{4}$ & $\mathrm{C}_{19} \mathrm{H}_{16} \mathrm{ClN}_{5} \mathrm{O}_{5} \mathrm{~S}$ & 461 & 122 & 75 & 49.41 & 3.49 & 15.16 \\
\hline 32 & 6dviii & $-\mathrm{OCH}_{3}$ & $-\mathrm{CH}=\mathrm{CH}-\mathrm{C}_{6} \mathrm{H}_{5}$ & $\mathrm{C}_{21} \mathrm{H}_{19} \mathrm{ClN}_{4} \mathrm{O}_{3} \mathrm{~S}$ & 442 & 140 & 54 & 56.95 & 4.32 & 12.65 \\
\hline
\end{tabular}




\section{COMPUTATIONAL METHODS}

\subsection{Selection of PDB Structure:}

The protein data bank (PDB) is a collection of crystal structures for proteins with bound ligands and co-activators. The X-ray crystal structure published by Stewart, et al. of the human DNA topoisomerase $(70 \mathrm{Kda})$ in complex with the camptothecin and covalent complex with A 22 base pair DNA duplex (PDB ID: 1T8I) were retrieved from protein data bank [6] based on good resolution and Ramachandran's plot analysis. The structure was chosen because of its high resolution $3.0 \AA$ compared to other available structures. In the Ramachandran's plot analysis, the residues were classified according to their regions in the quadrangle, which shows human DNA topoisomerase (1T8I) is having $87.3 \%$ residues in most favorable region and there is no residue in disallowed region.

\subsection{Ligand generation and Optimization:}

The chemically synthesized thiazolidinone and azetidinone series derivatives were sketched using ACD/ ChemSketch (12.0) software and saved in mol file format. The saved ligand compounds were later imported in MOE and refined using a systematic conformer search followed by geometry optimization and energy minimization of the lowest energy structure using the Merck Molecular Force Field (MMFF94) [9]. The individual compounds were finally saved in mol file format for further binding studies.

\subsection{Docking Algorithms:}

Here we report the use of MOE-Dock by Chemical Computing Group Inc. [10], which has the advantage flexible docking as well as integration with a graphical interface as well as with other modules, such as analysis, molecular mechanics, and molecular dynamics. Macromolecular crystallographic data, when available, can be a valuable source of information for discovering active ligands. MOE provides a collection of applications for visualizing and understanding details of receptor active sites and receptorligand interactions. These applications are used to suggest improvements to ligands or screen ligand databases for candidate binders. MOE-Dock utilizes a Monte Carlo simulated annealing process for docking a substrate into the active site of a macromolecule.

\subsection{Docking Simulations:}

\subsubsection{London dG Dock:}

In MOE London dG scoring is used as default setting to calculate the exact confirmation and configuration of the ligand to find the best molecule with minimum binding energy [11] and it can be used to develop potential drug molecules against the disease. The London dG scoring function estimates the free energy $\Delta \mathrm{G}$ of binding of the ligand from a given pose.

\subsection{Active Site Detection \& Visualization:}

Detection of candidate protein-ligand and protein-protein binding sites using a fast geometric algorithm is based on Edelsbrunner's alpha shapes [12]. Each site on a macromolecular structure is ranked according to its accessible hydrophobic contact surface. Visualization of individual sites or populate them with "dummy atoms" is used for docking calculations or starting points for de novo ligand design efforts [13].

\section{RESULTS AND DISCUSSION}

\subsection{Docking Study:}

In order to determine the binding mode of action of the most potent DNA topo I inhibitor, we performed a docking study. Since the main goal of this study was to perform docking to understand binding between receptor and 64 thiazolidinone and azetidinone series derivatives to get fruitful results. During docking most of the default settings were applied except that the number of Retain was 10 instead of 30 during docking in MOE. Protein structures were first repaired and then appropriately protonated in the presence of ligand using the Protonate3D [14] process in MOE. Proteins prepared in this manner were applied directly for docking. It is well documented in literature [15] that if a crystallographic structure of the protein complexed with a relatively close analog of the ligand is available, "ligand-based docking" may be performed. In this procedure, one or more conformations of the candidate ligand are fitted to the crystallographic structure of the known ligand by optimizing the similarity in electrostatic and steric potentials. The experimental structure of the "template" ligand is then deleted, leaving the candidate ligand docked to the protein. In addition, the conformation of the fitted ligand may be simultaneously optimized during the fitting. The same strategy was used to get best docking results. The default procedure using Triangle Matcher placement method with London dG scoring was used for the docking runs.<smiles>[R]c1ccc2nc(NCC(=O)NN3C(=O)SCC3([Al])[AlH2])sc2c1</smiles>

Table IV: London dG scoring of all 32 Thiazolidinone ligand compounds

\begin{tabular}{|c|c|c|c|c|}
\hline $\begin{array}{l}\text { S.N } \\
\text { o. }\end{array}$ & $\begin{array}{c}\text { Com } \\
\text { pou } \\
\text { nd }\end{array}$ & $\mathbf{R}$ & $\mathbf{A r}$ & $\begin{array}{l}\text { London dG } \\
\text { Scoring }\end{array}$ \\
\hline 1 & 5 ai & $-\mathrm{Cl}$ & $-\mathrm{C}_{6} \mathrm{H}_{5}$ & -8.9475 \\
\hline 2 & 5aii & $-\mathrm{Cl}$ & $-4-\mathrm{OCH}_{3} \mathrm{C}_{6} \mathrm{H}_{4}$ & -8.1289 \\
\hline 3 & 5aiii & $-\mathrm{Cl}$ & $\begin{array}{l}-4- \\
\mathrm{N}\left(\mathrm{CH}_{3}\right)_{2} \mathrm{C}_{6} \mathrm{H}_{4}\end{array}$ & -8.6394 \\
\hline 4 & 5aiv & $-\mathrm{Cl}$ & $-4-\mathrm{ClC}_{6} \mathrm{H}_{4}$ & -8.8501 \\
\hline 5 & $5 \mathrm{av}$ & $-\mathrm{Cl}$ & $\begin{array}{l}-3,4- \\
d i \mathrm{OCH}_{3} \mathrm{C}_{6} \mathrm{H}_{3}\end{array}$ & -9.1008 \\
\hline 6 & 5avi & $-\mathrm{Cl}$ & $-2-\mathrm{OH} \mathrm{C} \mathrm{H}_{4}$ & -12.8472 \\
\hline 7 & 5avii & $-\mathrm{Cl}$ & $-3-\mathrm{NO}_{2} \mathrm{C}_{6} \mathrm{H}_{4}$ & -8.8318 \\
\hline 8 & $\begin{array}{c}\text { 5avii } \\
\mathrm{i}\end{array}$ & $-\mathrm{Cl}$ & $\begin{array}{l}-\mathrm{CH}=\mathrm{CH}- \\
\mathrm{C}_{6} \mathrm{H}_{5}\end{array}$ & -8.7508 \\
\hline 9 & 5 bi & $-\mathrm{F}$ & $-\mathrm{C}_{6} \mathrm{H}_{5}$ & -9.1867 \\
\hline 10 & 5 bii & $-\mathrm{F}$ & $-4-\mathrm{OCH}_{3} \mathrm{C}_{6} \mathrm{H}_{4}$ & -10.5462 \\
\hline
\end{tabular}




\begin{tabular}{|c|c|c|c|c|}
\hline 11 & 5 biii & $-\mathrm{F}$ & $\begin{array}{l}-4- \\
\mathrm{N}\left(\mathrm{CH}_{3}\right)_{2} \mathrm{C}_{6} \mathrm{H}_{4}\end{array}$ & -11.0780 \\
\hline 12 & 5biv & $-\mathrm{F}$ & $-4-\mathrm{ClC}_{6} \mathrm{H}_{4}$ & -8.9076 \\
\hline 13 & $5 \mathrm{bv}$ & $-\mathrm{F}$ & $\begin{array}{l}-3,4- \\
d i \mathrm{OCH}_{3} \mathrm{C}_{6} \mathrm{H}_{3}\end{array}$ & -10.0663 \\
\hline 14 & 5 bvi & $-\mathrm{F}$ & $-2-\mathrm{OH} \mathrm{C} \mathrm{C}_{6} \mathrm{H}_{4}$ & -11.6120 \\
\hline 15 & $\begin{array}{c}5 \text { bvi } \\
\text { i }\end{array}$ & $-\mathrm{F}$ & $-3-\mathrm{NO}_{2} \mathrm{C}_{6} \mathrm{H}_{4}$ & -9.7053 \\
\hline 16 & $\begin{array}{l}\text { bbvi } \\
\text { ii }\end{array}$ & $-\mathrm{F}$ & $\begin{array}{l}-\mathrm{CH}=\mathrm{CH}- \\
\mathrm{C}_{6} \mathrm{H}_{5}\end{array}$ & -9.1136 \\
\hline 17 & $5 \mathrm{ci}$ & $-\mathrm{CH}_{3}$ & $-\mathrm{C}_{6} \mathrm{H}_{5}$ & -9.0949 \\
\hline 18 & 5 cii & $-\mathrm{CH}_{3}$ & $-4-\mathrm{OCH}_{3} \mathrm{C}_{6} \mathrm{H}_{4}$ & -9.6069 \\
\hline 19 & 5 ciii & $-\mathrm{CH}_{3}$ & $\begin{array}{l}-4- \\
\mathrm{N}\left(\mathrm{CH}_{3}\right)_{2} \mathrm{C}_{6} \mathrm{H}_{4}\end{array}$ & -9.5630 \\
\hline 20 & 5 civ & $-\mathrm{CH}_{3}$ & $-4-\mathrm{ClC}_{6} \mathrm{H}_{4}$ & -9.7327 \\
\hline 21 & $5 \mathrm{cv}$ & $-\mathrm{CH}_{3}$ & $\begin{array}{l}-3,4- \\
d i \mathrm{OCH}_{3} \mathrm{C}_{6} \mathrm{H}_{3}\end{array}$ & -10.2671 \\
\hline 22 & $5 \mathrm{cvi}$ & $-\mathrm{CH}_{3}$ & $-2-\mathrm{OH} \mathrm{C} \mathrm{C}_{6} \mathrm{H}_{4}$ & -9.7411 \\
\hline 23 & 5 cvii & $-\mathrm{CH}_{3}$ & $-3-\mathrm{NO}_{2} \mathrm{C}_{6} \mathrm{H}_{4}$ & -9.1137 \\
\hline 24 & $\begin{array}{c}5 \text { cvii } \\
\text { i }\end{array}$ & $-\mathrm{CH}_{3}$ & $\begin{array}{l}-\mathrm{CH}=\mathrm{CH}- \\
\mathrm{C}_{6} \mathrm{H}_{5}\end{array}$ & -8.9317 \\
\hline 25 & $5 \mathrm{di}$ & $-\mathrm{OCH}_{3}$ & $-\mathrm{C}_{6} \mathrm{H}_{5}$ & -8.5772 \\
\hline 26 & 5 dii & $-\mathrm{OCH}_{3}$ & $-4-\mathrm{OCH}_{3} \mathrm{C}_{6} \mathrm{H}_{4}$ & -7.8430 \\
\hline 27 & 5diii & $-\mathrm{OCH}_{3}$ & $\begin{array}{l}-4- \\
\mathrm{N}\left(\mathrm{CH}_{3}\right)_{2} \mathrm{C}_{6} \mathrm{H}_{4}\end{array}$ & -9.0294 \\
\hline 28 & $5 \operatorname{div}$ & $-\mathrm{OCH}_{3}$ & $-4-\mathrm{ClC}_{6} \mathrm{H}_{4}$ & -9.3360 \\
\hline 29 & $5 \mathrm{dv}$ & $-\mathrm{OCH}_{3}$ & $\begin{array}{l}-3,4- \\
d i \mathrm{OCH}_{3} \mathrm{C}_{6} \mathrm{H}_{3}\end{array}$ & -9.2706 \\
\hline 30 & $5 \mathrm{dvi}$ & $-\mathrm{OCH}_{3}$ & $-2-\mathrm{OH} \mathrm{C} \mathrm{C}_{6} \mathrm{H}_{4}$ & -9.1197 \\
\hline 31 & $\begin{array}{c}5 \mathrm{dvi} \\
\mathrm{i}\end{array}$ & $-\mathrm{OCH}_{3}$ & $-3-\mathrm{NO}_{2} \mathrm{C}_{6} \mathrm{H}_{4}$ & -9.7540 \\
\hline 32 & $\begin{array}{l}5 \mathrm{dvi} \\
\text { ii }\end{array}$ & $-\mathrm{OCH}_{3}$ & $\begin{array}{l}-\mathrm{CH}=\mathrm{CH}- \\
\mathrm{C}_{6} \mathrm{H}_{5}\end{array}$ & -8.4168 \\
\hline
\end{tabular}

\begin{tabular}{|c|c|c|c|c|}
\hline 14 & 6bvi & $-\mathrm{F}$ & $-2-\mathrm{OH} \mathrm{C} \mathrm{C}_{6} \mathrm{H}_{4}$ & -9.6012 \\
\hline 15 & 6bvii & $-\mathrm{F}$ & $-3-\mathrm{NO}_{2} \mathrm{C}_{6} \mathrm{H}_{4}$ & -9.7504 \\
\hline 16 & $\begin{array}{c}\text { 6bvii } \\
\text { i }\end{array}$ & $-\mathrm{F}$ & $\begin{array}{l}-\mathrm{CH}=\mathrm{CH}- \\
\mathrm{C}_{6} \mathrm{H}_{5}\end{array}$ & -9.2131 \\
\hline 17 & $6 \mathrm{ci}$ & $-\mathrm{CH}_{3}$ & $-\mathrm{C}_{6} \mathrm{H}_{5}$ & -8.2349 \\
\hline 18 & $6 \mathrm{cii}$ & $-\mathrm{CH}_{3}$ & $-4-\mathrm{OCH}_{3} \mathrm{C}_{6} \mathrm{H}_{4}$ & -9.9519 \\
\hline 19 & 6ciii & $-\mathrm{CH}_{3}$ & $\begin{array}{l}-4- \\
\mathrm{N}\left(\mathrm{CH}_{3}\right)_{2} \mathrm{C}_{6} \mathrm{H}_{4}\end{array}$ & -9.2802 \\
\hline 20 & $6 \mathrm{civ}$ & $-\mathrm{CH}_{3}$ & $-4-\mathrm{ClC}_{6} \mathrm{H}_{4}$ & -9.6475 \\
\hline 21 & $6 \mathrm{cv}$ & $-\mathrm{CH}_{3}$ & $\begin{array}{l}-3,4- \\
d i \mathrm{OCH}_{3} \mathrm{C}_{6} \mathrm{H}_{3}\end{array}$ & -10.4800 \\
\hline 22 & $6 \mathrm{cvi}$ & $-\mathrm{CH}_{3}$ & $-2-\mathrm{OH} \mathrm{C}{ }_{6} \mathrm{H}_{4}$ & -11.0270 \\
\hline 23 & 6 cvii & $-\mathrm{CH}_{3}$ & $-3-\mathrm{NO}_{2} \mathrm{C}_{6} \mathrm{H}_{4}$ & -9.6658 \\
\hline 24 & $\begin{array}{c}6 c v i i \\
\text { i }\end{array}$ & $-\mathrm{CH}_{3}$ & $\begin{array}{l}-\mathrm{CH}=\mathrm{CH}- \\
\mathrm{C}_{6} \mathrm{H}_{5}\end{array}$ & -8.9973 \\
\hline 25 & 6di & $-\mathrm{OCH}_{3}$ & $-\mathrm{C}_{6} \mathrm{H}_{5}$ & -8.4538 \\
\hline 26 & 6dii & $-\mathrm{OCH}_{3}$ & $-4-\mathrm{OCH}_{3} \mathrm{C}_{6} \mathrm{H}_{4}$ & -8.7592 \\
\hline 27 & 6diii & $-\mathrm{OCH}_{3}$ & $\begin{array}{l}-4- \\
\mathrm{N}\left(\mathrm{CH}_{3}\right)_{2} \mathrm{C}_{6} \mathrm{H}_{4}\end{array}$ & -8.5255 \\
\hline 28 & 6div & $-\mathrm{OCH}_{3}$ & $-4-\mathrm{ClC}_{6} \mathrm{H}_{4}$ & -9.4972 \\
\hline 29 & $6 \mathrm{dv}$ & $-\mathrm{OCH}_{3}$ & $\begin{array}{l}-3,4- \\
d i \mathrm{OCH}_{3} \mathrm{C}_{6} \mathrm{H}_{3}\end{array}$ & -8.7395 \\
\hline 30 & $6 \mathrm{dvi}$ & $-\mathrm{OCH}_{3}$ & $-2-\mathrm{OH} \mathrm{C} \mathrm{C}_{6}$ & -9.8413 \\
\hline 31 & 6dvii & $-\mathrm{OCH}_{3}$ & $-3-\mathrm{NO}_{2} \mathrm{C}_{6} \mathrm{H}_{4}$ & -10.6481 \\
\hline 32 & $\begin{array}{c}\text { 6dvii } \\
\text { i }\end{array}$ & $-\mathrm{OCH}_{3}$ & $\begin{array}{l}-\mathrm{CH}=\mathrm{CH}- \\
\mathrm{C}_{6} \mathrm{H}_{5}\end{array}$ & -8.6431 \\
\hline
\end{tabular}

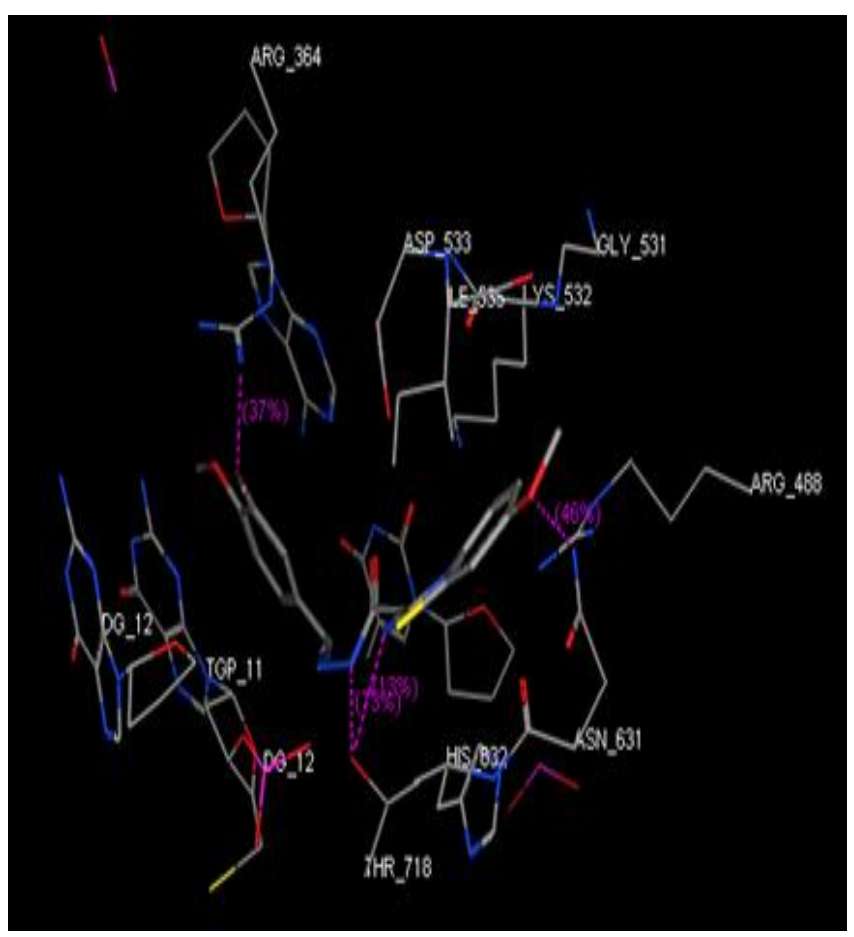

Table V: London dG scoring of all 32 Azetidinone ligand compounds

\begin{tabular}{|c|c|l|l|c|}
\hline $\begin{array}{c}\text { S.N } \\
\text { o. }\end{array}$ & $\begin{array}{c}\text { Com } \\
\text { poun } \\
\text { d }\end{array}$ & \multicolumn{1}{|c|}{$\mathbf{R}$} & \multicolumn{1}{|c|}{$\mathbf{A r}$} & $\begin{array}{c}\text { London dG } \\
\text { Scoring }\end{array}$ \\
\hline 1 & 6 ai & $-\mathrm{Cl}$ & $-\mathrm{C}_{6} \mathrm{H}_{5}$ & -8.8295 \\
\hline 2 & 6 aii & $-\mathrm{Cl}$ & $-4-\mathrm{OCH}_{3} \mathrm{C}_{6} \mathrm{H}_{4}$ & -8.5629 \\
\hline 3 & 6 aiii & $-\mathrm{Cl}$ & $\begin{array}{l}-4- \\
\mathrm{N}\left(\mathrm{CH}_{3}\right)_{2} \mathrm{C}_{6} \mathrm{H}_{4}\end{array}$ & -10.4502 \\
\hline 4 & 6 aiv & $-\mathrm{Cl}$ & $-4-\mathrm{ClC}_{6} \mathrm{H}_{4}$ & -8.8006 \\
\hline 5 & 6 av & $-\mathrm{Cl}$ & $\begin{array}{l}-3,4- \\
\text { di } \mathrm{OCH}_{3} \mathrm{C}_{6} \mathrm{H}_{3}\end{array}$ & -9.1750 \\
\hline 6 & 6 avi & $-\mathrm{Cl}$ & $-2-\mathrm{OH} \mathrm{C}_{6} \mathrm{H}_{4}$ & -9.2040 \\
\hline 7 & 6 avii & $-\mathrm{Cl}$ & $-3-\mathrm{NO}_{2} \mathrm{C}_{6} \mathrm{H}_{4}$ & -8.7993 \\
\hline 8 & 6 avii & $-\mathrm{Cl}$ & $\begin{array}{l}-\mathrm{CH}_{2} \mathrm{CH}_{-} \\
\mathrm{C}_{6} \mathrm{H}_{5}\end{array}$ & -7.8580 \\
\hline 9 & i bi & $-\mathrm{F}$ & $-\mathrm{C}_{6} \mathrm{H}_{5}$ & -8.8573 \\
\hline 10 & 6 bii & $-\mathrm{F}$ & $-4-\mathrm{OCH}_{3} \mathrm{C}_{6} \mathrm{H}_{4}$ & -9.3875 \\
\hline 11 & 6 biii & $-\mathrm{F}$ & $\begin{array}{l}-4- \\
\mathrm{N}\left(\mathrm{CH}_{3}\right)_{2} \mathrm{C}_{6} \mathrm{H}_{4}\end{array}$ & -10.229 \\
\hline 12 & $6 \mathrm{biv}$ & $-\mathrm{F}$ & $-4-\mathrm{ClC}_{6} \mathrm{H}_{4}$ & -9.2295 \\
\hline 13 & $6 \mathrm{bv}$ & $-\mathrm{F}$ & $\begin{array}{l}-3,4- \\
\text { diOCH}\end{array} \mathrm{C}_{6} \mathrm{H}_{3}$ & -8.9526 \\
\hline
\end{tabular}

\section{Fig 1: H-bond interactions of Compound 5avi with active} site residues of Human DNA Topo I

From the docking it is clear that the side chain and backbone of residue surrounding the active site adjust to each bound drug with each drug by different amount this suggests that the conformation of receptor changes on introduction of drug at active site. This means this protein is able to interact and accommodate inhibitors of different chemical structures. The results demonstrate that thiazolidinone and azetidinone series 
derivatives can be docked and aligned into the DNA topoisomerase allosteric binding site extremely well. The docking studies reveal that the most active compound 5avi from thiazolidinone derivate and $6 \mathrm{cvi}$ from azetidinone binds to protein not only due to hydrogen bonding but due to hydrophobic and electrostatic interactions also. Table III contains docking scores of all 32 thiazolidinone ligands and Table IV contains docking scores of all 32 azetidinone ligands. The best ligand was chosen on the basis of high London dG Scoring and highly interacting amino acid residues. From each of 32 thiazolidinone and azetidinone series derivatives the compound 5avi shows high binding score and the interactions of the compound with the protein is shown in figure 1 . The ligand poses were analyzed and interaction of ligand molecule with the 1T8I protein structure was studied on the basis of H-bonding made by the poses to the receptor molecule and close contacts (Vander Waals clashes) between the poses and receptor molecule. As it is well known, $\mathrm{H}$ bonds play an important role for the structure and function of biological molecules, especially for the enzyme catalysis.

\section{CONCLUSION}

In the present study, each of 32 thiazolidinone and azetidinone series derivatives were docked in active site of Human DNA Topoisomerase I enzyme. From the docking analysis, the compounds 5avi of thiazolidene and $6 \mathrm{cvi}$ of azetidinone derivative shows high binding affinity with the receptor. Further careful inspection of the binding pocket indicated the compound 5avi shows H-bond interactions with active site residues of Human DNA Topoisomerase I such as DT10, TGP11, DG112, DA113, ASN352, Glu356, Lys374, Lys425 and the compound 6cvi shows interactions with DT10, TGP11, Glu 356, lys425, Arg 4888. Hence, compound 5avi of the thiazolidinone series and $6 \mathrm{cvi}$ of azetidinone series can be assumed to be the most potent topo I inhibitor and can be potentially act as anti-cancer target.

\section{ACKNOWLEDGEMENTS}

The authors are thankful for the facilitation provided by Vikas College of Pharmacy, Jangaon and Asterace Labs, Hyderabad in conducting this study.

\section{REFERENCES}

[1] Wang, J. C. Annu. Rev. Biochem. 1996, 65, 635; (b) Liu, L. F. Annu. Rev. Biochem. 1989, 58, 351.
[2] Liew, S. T.; Yang, L. X. Curr. Pharm. Des. 2008, 14, 1078.

[3] Takagi, K.; Dexheimer, T. S.; Redon, C.; Sordet, O.; Agama, K.; Lavielle, G.; Pierre, A.; Bates, S. E.; Pommier, Y. Mol. Cancer Ther. 2007, 6, 3229; (b) Bjornsti, M. A.; Benedetti, P.; Viglianti, G. A.; Wang, J. C. Cancer Res. 1989, 49, 6318; (c) Hsiang, Y. H.; Liu, L. F. Cancer Res. 1988, 48, 1722.

[4] Pommier, Y. Biochimie 1998, 80, 255.

[5] Pommier, Y.; Pourquier, P.; Urasaki, Y.; Wu, J.; Laco, G. S. Drug Resist. Updates 1999, 2, 307.

[6] http://www.rcsb.org/pdb

[7] Merabet, N.; Dumond, J.; Collinet, B.; Van Baelinghem, L.; Boggetto, N.; Ongeri, S.; Ressad, F.; Reboud-Ravaux, M.; Sicsic, S. J. Med. Chem. 2004, 47, 6392.

[8] Reference Ai Jeng and Sudhaka Kasina. Synthesis of 3substituted 7-(3,3-dimethyl-1-triazeno)-10methylphenothiazines as potential antitumor agents. J. Heterocyclic Chem.1981; 18,759.

[9] Halgren, T. J. Comput. Chem. 1996, 17, 490.

[10] MOE Molecular Operating Environment, Version 2008; Chemical Computing Group Inc.:Montreal, Canada, 2008.

[11] E. DeClercq, A. Karlsson, J. Balzarini, In; E. A. Rekka P. N. Kourounakis, Chemistry And Molecular Aspects Of Drug Design And Action, CRC Press Taylor \& Francis Group, LLC, 2008, pp177-198.

[12] Edelsbrunner, H., Facello, M., Fu, R., Liang, J. Measuring Proteins and Voids in Proteins. Proceedings of the 28th Annual Hawaii International Conference on Systems Science. 256-264 (1995).

[13] Goodford, P.J. A Computational Procedure for Determining Energetically Favorable Binding Sites on Biologically Important Macromolecules. J. Med. Chem.28, No. 7, 849-857 (1985).

[14] H. Singh, S. S. Marla, M. Agarwal, IAENG Int. J. Comp. Sci., 2007, 33(1), 1-7.

[15] N. C. Cohen, Guidebook on Molecular modeling in Drug Design, Academic Press, 1996, 253-257. 